\title{
Prediabetes and risk of mortality, diabetes-related complications and comorbidities: umbrella review of meta-analyses of prospective studies
}

\author{
Sabrina Schlesinger ${ }^{1,2}$ (D) $\cdot$ Manuela Neuenschwander ${ }^{1,2}$ (D) - Janett Barbaresko ${ }^{1}$ (D) $\cdot$ Alexander Lang ${ }^{1} \cdot$ \\ Haifa Maalmi ${ }^{2,3}$ (i) $\cdot$ Wolfgang Rathmann ${ }^{1,2}$ (1) $\cdot$ Michael Roden ${ }^{2,3,4}$ (I) $\cdot$ Christian Herder ${ }^{2,3,4}$ (i)
}

Received: 26 April 2021 / Accepted: 10 August 2021 / Published online: 31 October 2021

(C) The Author(s) 2021

\begin{abstract}
Aims/hypothesis The term prediabetes is used for individuals who have impaired glucose metabolism whose glucose or $\mathrm{Hb}_{1 \mathrm{c}}$ levels are not yet high enough to be diagnosed as diabetes. Prediabetes may already be associated with an increased risk of chronic 'diabetes-related' complications. This umbrella review aimed to provide a systematic overview of the available evidence from meta-analyses of prospective observational studies on the associations between prediabetes and incident diabetes-related complications in adults and to evaluate their strength and certainty.

Methods For this umbrella review, systematic reviews with meta-analyses reporting summary risk estimates for the associations between prediabetes (based on fasting or $2 \mathrm{~h}$ postload glucose or on $\mathrm{HbA}_{1 \mathrm{c}}$ ) and incidence of diabetes-related complications, comorbidities and mortality risk were included. PubMed, Web of Science, the Cochrane Library and Epistemonikos were searched up to 17 June 2021. Summary risk estimates were recalculated using a random effects model. The certainty of evidence was evaluated by applying the GRADE tool. This study is registered with PROSPERO, CRD42020153227.

Results Ninety-five meta-analyses from 16 publications were identified. In the general population, prediabetes was associated with a 6-101\% increased risk for all-cause mortality and the incidence of cardiovascular outcomes, CHD, stroke, heart failure, atrial fibrillation and chronic kidney disease, as well as total cancer, total liver cancer, hepatocellular carcinoma, breast cancer and all-cause dementia with moderate certainty of evidence. No associations between prediabetes and incident depressive symptoms and cognitive impairment were observed (with low or very low certainty of evidence). The association with all-cause mortality was stronger for prediabetes defined by impaired glucose tolerance than for prediabetes defined by $\mathrm{HbA}_{1 \mathrm{c}}$.

Conclusions/interpretation Prediabetes was positively associated with risk of all-cause mortality and the incidence of cardiovascular outcomes, CHD, stroke, chronic kidney disease, cancer and dementia. Further high-quality studies, particularly on $\mathrm{HbA}_{1 \mathrm{c}}$-defined prediabetes and other relevant health outcomes (e. g. neuropathy) are required to support the evidence.
\end{abstract}

Keywords Complications $\cdot$ Meta-analysis $\cdot$ Mortality $\cdot$ Prediabetes $\cdot$ Systematic review $\cdot$ Umbrella review

Sabrina Schlesinger

sabrina.schlesinger@ddz.de

1 Institute for Biometrics and Epidemiology, German Diabetes Center (Deutsches Diabetes-Zentrum/DDZ), Leibniz Center for Diabetes Research at Heinrich Heine University Düsseldorf, Düsseldorf, Germany

2 German Center for Diabetes Research (DZD), Partner Düsseldorf, Düsseldorf, Germany
3 Institute for Clinical Diabetology, German Diabetes Center (Deutsches Diabetes-Zentrum/DDZ), Leibniz Center for Diabetes Research at Heinrich Heine University Düsseldorf, Düsseldorf, Germany

4 Department of Endocrinology and Diabetology, Medical Faculty and University Hospital, Heinrich Heine University, Düsseldorf, Germany 


\section{Research in context}

\section{What is already known about this subject?}

- The term prediabetes is used for individuals with impaired glucose metabolism (based on fasting or $2 \mathrm{~h}$ postload glucose or on $\mathrm{HbA}_{1 c}$ ) whose glucose or $\mathrm{HbA}_{1 c}$ levels are not high enough yet to be diagnosed as type 2 diabetes

- The global prevalence of prediabetes is increasing steadily but its implications for the risk of comorbidities and other disorders that are traditionally considered as complications of diabetes are uncertain

- A comprehensive systematic overview of the available evidence and its certainty and the influence of potential bias for associations between prediabetes and 'diabetes-related' complications is needed

\section{What is the key question?}

- Is prediabetes associated with health outcomes, and how strong is the certainty of evidence?

\section{What are the new findings?}

- There is moderate certainty of evidence that prediabetes is associated with an increased risk for all-cause mortality and the incidence of cardiovascular events, CHD, stroke, heart failure, atrial fibrillation, chronic kidney disease, cancer and dementia, compared with people without prediabetes

- A lack of association was observed for prediabetes and incidence of depression or cognitive disorders, with low or very low certainty of evidence

- No meta-analyses on common and clinically relevant complications, such as heart failure, peripheral and autonomic neuropathy and retinopathy, were identified

\section{How might this impact on clinical practice in the foreseeable future?}

- Given the high global prevalence of prediabetes and the potential health benefits of lifestyle interventions in people with prediabetes, future studies in this field using state-of-the-art methodologies and adhering to reporting guidelines should be made research priorities

\section{Abbreviations \\ IFG Impaired fasting glucose \\ IGT Impaired glucose tolerance \\ SHR Summary HR}

\section{Introduction}

The term 'prediabetes' is used for individuals with impaired glucose metabolism not fulfilling the diagnostic criteria of type 2 diabetes. Based on the recent guidelines from the ADA, this definition is met if at least one of the following criteria applies: impaired fasting glucose (IFG) (fasting plasma glucose 5.6-6.9 mmol/l); impaired glucose tolerance (IGT) (2 h glucose during a $75 \mathrm{~g}$ OGTT $7.8-11.0 \mathrm{mmol} / \mathrm{l}$ ); or $\mathrm{HbA}_{1 \mathrm{c}}$ in the range of $39-47 \mathrm{mmol} / \mathrm{mol}(5.7-6.4 \%)$ [1]. The IDF estimated the prevalence of IGT in the adult population to be $7.5 \%$ (373.9 million) in 2019 and projected an increase to $8.6 \%$ (548.4 million) in 2045 [2]. A recent study in China used fasting plasma glucose and $\mathrm{HbA}_{1 \mathrm{c}}$ to define prediabetes and found a prevalence of $35.7 \%$ among the Chinese adult population [3].

The concept of prediabetes is important because of its high prevalence and the high risk of progression to overt type 2 diabetes among individuals with prediabetes $[1,4]$. However, various metabolic abnormalities already exist before the onset of diabetes [5] and may confer an increased risk of multiple comorbidities and chronic complications that are traditionally seen as 'diabetes-related' [2,6]. Metaanalyses have linked prediabetes with higher risk of cardiovascular outcomes and chronic kidney disease [7, 8]. In addition, there is emerging evidence that prediabetes is associated with the development of less frequently assessed comorbidities of diabetes such as cognitive disorders and cancer $[9,10]$. A recent study from the UK estimated that half of the study population already had a macro- or microvascular disease at the time of type 2 diabetes diagnosis [11].

Given these findings, prediabetes represents a time window of opportunity in which modifiable risk factors, such as overweight/obese state, diet high in energy and physical inactivity, can be targeted to prevent or delay the development of type 2 diabetes $[12,13]$. Lifestyle intervention in people with prediabetes may also have beneficial effects on the 
Table 1 Definitions of prediabetes

\begin{tabular}{lccc}
\hline Definition & $\begin{array}{l}\text { ADA } \\
\text { Prediabetes }\end{array}$ & $\begin{array}{l}\text { WHO } \\
\text { Intermediate hyperglycaemia }\end{array}$ & $\begin{array}{l}\text { IDF } \\
\text { IFG, IGT }\end{array}$ \\
\hline IFG & & & $6.1-6.9$ \\
FPG in mmol/1 & $5.6-6.9$ & $6.1-6.9$ & $110-125$ \\
FPG in mg/dl & $100-125$ & $110-125$ & $7.8-11.0$ \\
IGT & & & $140-199$ \\
$2 \mathrm{~h} \mathrm{PG}$ in mmol/l & $7.8-11.0$ & $7.8-11.0$ & - \\
$2 \mathrm{~h} \mathrm{PG}$ in mg/dl & $140-199$ & $140-199$ & - \\
$\mathrm{HbA}$ & & & - \\
$\mathrm{mmol} / \mathrm{mol}$ & $39-47$ & & \\
$\%$ & $5.7-6.4$ & & \\
\hline
\end{tabular}

$2 \mathrm{~h}$ PG, 2 h plasma glucose during 75 g OGTT; FPG, fasting plasma glucose development of CVD, microvascular complications and both cardiovascular and all-cause mortality in the long-term [14]. However, this is controversial given the lack of studies with appropriate sample sizes and follow-up times [15].

To estimate the full clinical relevance of prediabetes as a potential indication for intervention measures, clarification is needed regarding the health outcomes for which prediabetes is a risk factor and the strength of evidence for these associations. In addition, potential biases of the published reports and research gaps in the literature need to be evaluated. In this context, umbrella reviews are helpful tools because they provide a broad overview of the existing evidence by focusing on published systematic reviews with meta-analyses on a specific topic and enable evaluation of the certainty of evidence and the risk of bias of the published meta-analyses [16].

Therefore, this study aimed to conduct an umbrella review of systematic reviews with meta-analyses to provide a comprehensive overview of the available evidence from prospective studies on the association between prediabetes and the incidence of diabetes-related complications and comorbidities in adults and to evaluate the strength and certainty of this evidence.

\section{Methods}

Data sources and searches This umbrella review was conducted and reported according to the PRISMA statement and to 'Preferred reporting items for overviews of systematic reviews' $[17,18]$. A protocol was prospectively registered at PROSPERO (CRD42020153227).

The systematic literature search was conducted from inception up to 17 June 2021 in PubMed, Web of Science, the Cochrane Library and Epistemonikos by using a pre-defined search term (electronic supplementary material [ESM] Table 1). We did not apply any restrictions or filters. To identify further relevant articles, we screened the reference lists of the identified reports. The literature screening was conducted independently by at least two authors. Disagreements were resolved by consensus.

Study selection Systematic reviews with meta-analyses from prospective studies on prediabetes and incidence of diabetesrelated complications, comorbidities or risk of mortality were included if they met the following eligibility criteria: prediabetes had to be defined according to blood glucose or $\mathrm{HbA}_{1 \mathrm{c}}$ levels that were higher than normal but not high enough to be diagnosed as diabetes. We accepted the diagnostic criteria from the ADA, WHO and IDF (Table 1). The comparators were defined as no prediabetes according to blood glucose levels or $\mathrm{HbA}_{1 \mathrm{c}}$ in the normal range. We only considered meta-analyses that were based on the following inclusion criteria: adult participants ( $\geq 18$ years); study sample from the general population or patient groups (e.g. with CVD); and absence of diagnosed diabetes or gestational diabetes at baseline. Meta-analyses on children, adolescents or pregnant women were excluded (we are not aware of any meta-analyses or identified meta-analyses during our search that focused on these groups). Regarding the outcomes, we considered incidence of any diabetes-related complications, comorbidities or mortality risk without restrictions. Systematic reviews without a meta-analysis were excluded. If a report presented more than one meta-analysis (multiple outcomes [e.g. risk of all-cause mortality, CVD mortality, stroke, etc.]), all meta-analyses were included separately. If reports focused on the same outcome but used different prediabetes definitions, all reports were included as single meta-analyses. If multiple reports were available for the meta-analysis (same definition of prediabetes and same outcome), we selected the largest meta-analysis with the largest number of primary studies and/or the report that provided the most detailed information (e.g. 


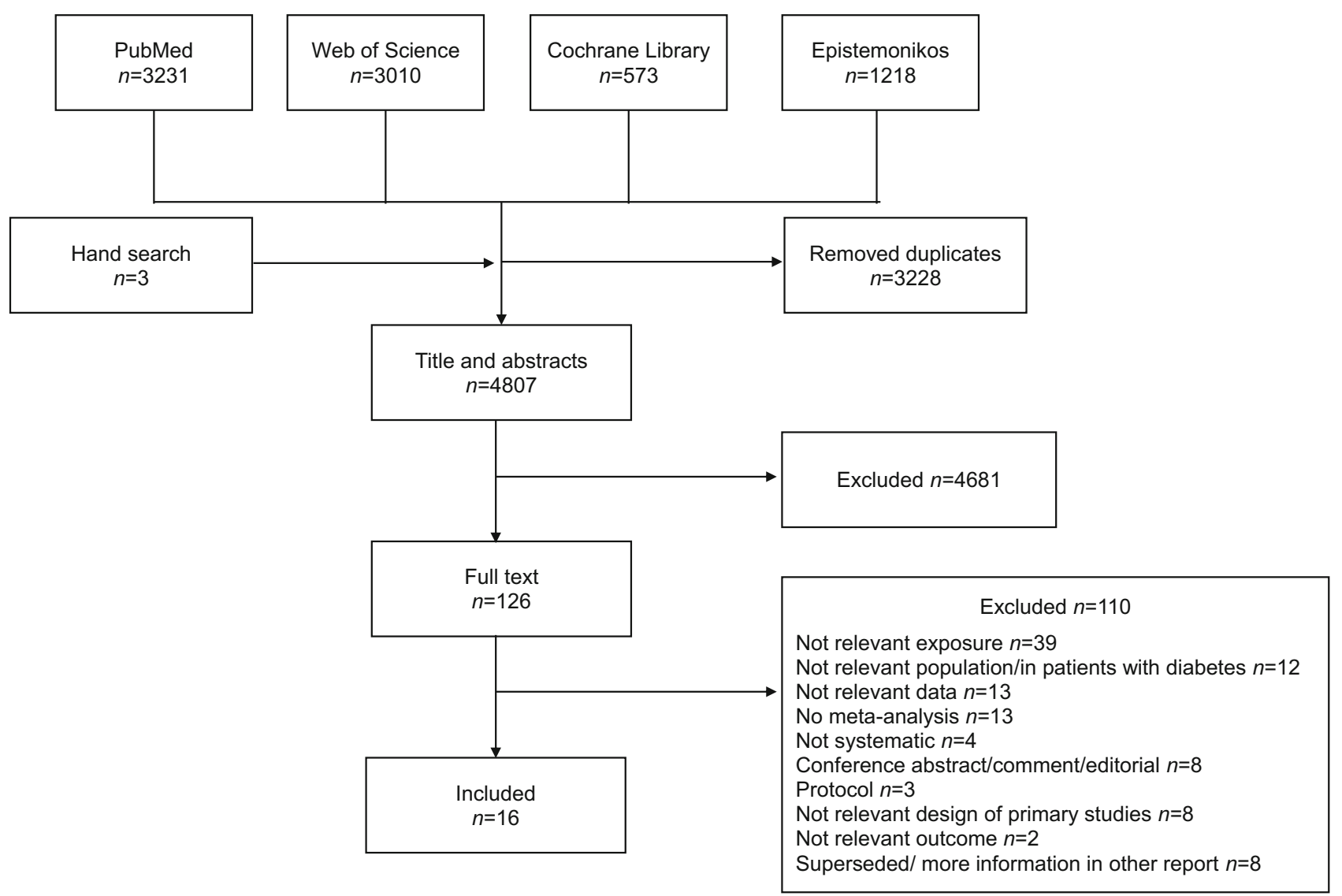

Fig. 1 Flow chart of the study selection process

meta-analyses separately for the general population and participant groups).

Data extraction and quality assessment One author extracted relevant data and another investigator double-checked extracted data for accuracy. Any discrepancies were discussed and resolved by discussion. For each published meta-analysis, the data listed in ESM Table 2 were extracted.

The methodological quality was evaluated by applying the ROBIS tool [19]. This tool consists of four domains: (1) study eligibility criteria; (2) identification and selection of studies; (3) data collection and study appraisal; and (4) synthesis and findings. Finally, an overall evaluation (high or low risk of bias) considering the identified bias along with the interpretation of findings and discussion of the limitations was done. At least two researchers independently assessed the risk of bias of each meta-analysis, and discrepancies were resolved by discussion.

Data synthesis and analysis The summary HRs (SHRs) and their corresponding 95\% CIs for the association of prediabetes (vs blood glucose levels or $\mathrm{HbA}_{1 \mathrm{c}}$ in the normal range) with diabetes-related complications were recalculated using the random effects model of DerSimonian and Laird [20]. When an original meta-analysis presented results from the same cohort separately (e.g. by sex), we combined the summary effects per cohort first, using a fixed effect model, before conducting the overall meta-analysis. If a published metaanalysis included a study with a prediabetes definition that was not in accordance with our definition, we excluded this primary study in our recalculations. In addition, if a published meta-analysis combined primary studies with different prediabetes definitions, we additionally recalculated this metaanalysis for each prediabetes definition separately whenever sufficient data were available. Heterogeneity was evaluated by calculating $I^{2}, \tau^{2}$ and $95 \%$ prediction intervals. Small study effects and publication bias were assessed by using graphical (funnel plots) and statistical tests (Egger's test), if $\geq 10$ studies were available [21]. A $p$ value of $<0.10$ indicated the presence of publication bias. All statistical analyses were performed using Stata (version 15; Stata-Corp, College Station, TX, USA).

Certainty of evidence The certainty of evidence was evaluated by two authors independently using the GRADE tool [22]. Observational studies start with a low certainty of evidence because of risk of bias due to residual confounding. Indications of inconsistency across studies (differences of point estimates, non-overlap of $95 \%$ CIs and $I^{2}$ statistics), indirectness (e.g. mixed outcomes), imprecision of 


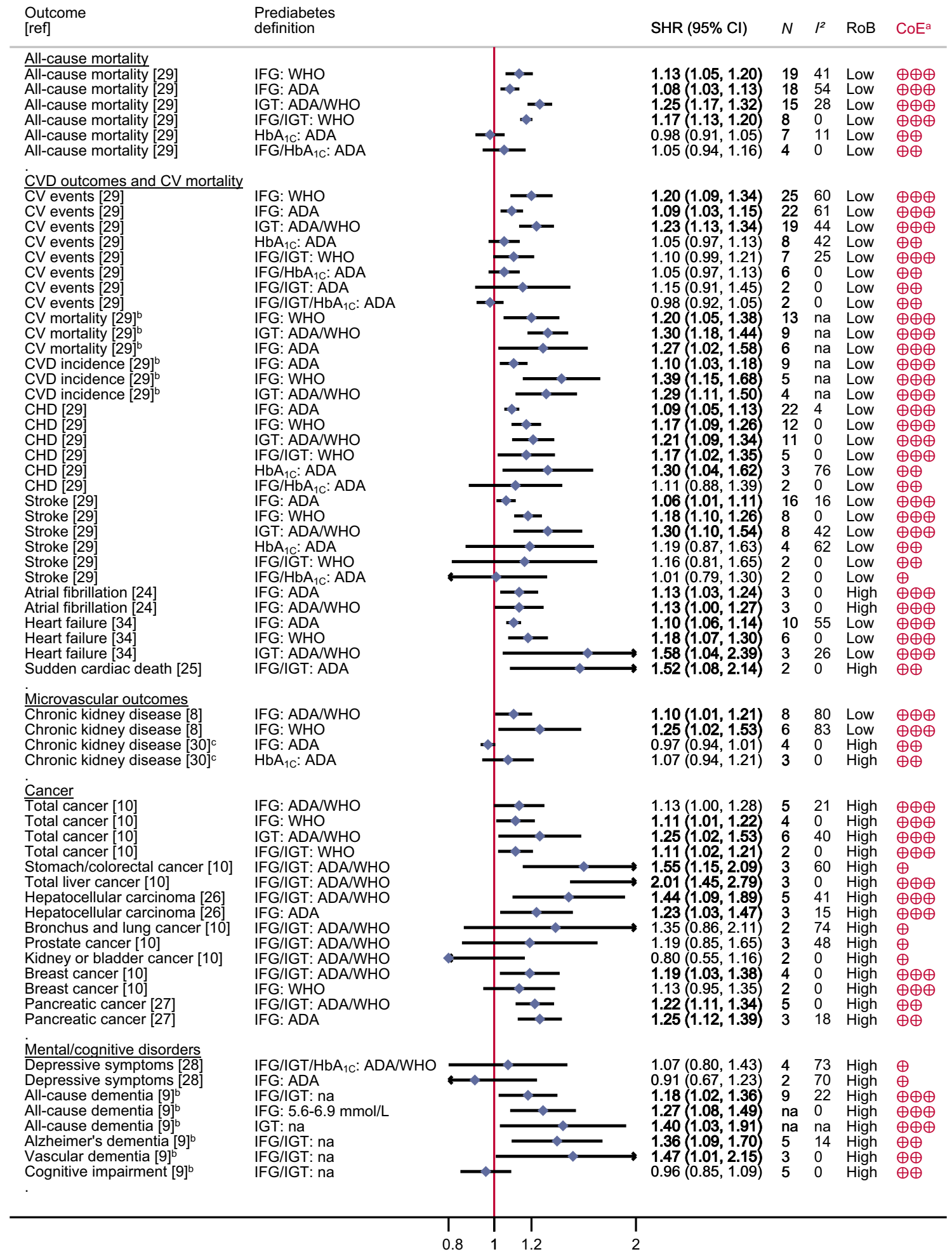

Fig. 2 Associations between prediabetes and risk of diabetes-related complications, comorbidities and mortality among the general population. Interpretation of the certainty of evidence is denoted by crossed circles: four symbols, high; three symbols, moderate; two symbols, low; and one symbol, very low. ${ }^{b}$ Could not be recalculated because of missing information. ${ }^{\mathrm{c}}$ Individuals with diabetes at follow-up were excluded. Boldface indicates that the $95 \%$ CI does not include the null value and findings are precisely estimated. $\mathrm{CoE}$, certainty of evidence; $\mathrm{CV}$, cardiovascular; $N$, number of primary studies; na, not available; RoB, risk of bias the findings (precision of the $95 \% \mathrm{CI}$ and the inclusion of appreciable benefit and harm) and the presence of publication bias can lead to downgrading. On the other hand, a large effect (SHR either $\geq 2.0$ or $\leq 0.5$ ) and a 


\begin{tabular}{|c|c|c|c|c|c|c|c|}
\hline \multirow{2}{*}{$\begin{array}{l}\begin{array}{l}\text { Outcome } \\
\text { [ref] }\end{array} \\
\text { All-cause mortality }\end{array}$} & \multicolumn{2}{|l|}{$\begin{array}{l}\text { Prediabetes } \\
\text { definition }\end{array}$} & \multirow[t]{2}{*}{ SHR $(95 \% \mathrm{Cl})$} & \multirow[t]{2}{*}{$N$} & \multirow[t]{2}{*}{$1^{2}$} & \multirow[t]{2}{*}{ RoB } & \multirow[t]{2}{*}{$\mathrm{CoE}^{\mathrm{a}}$} \\
\hline & & & & & & & \\
\hline All-cause mortality $[29]^{\mathrm{b}}$ & IFG: ADA & $\longrightarrow$ & $1.60(1.15,2.22)$ & 5 & 70 & Low & $\oplus \oplus$ \\
\hline All-cause mortality $[29]^{\mathrm{b}}$ & IFG: WHO & $-\infty$ & $1.19(0.98,1.45)$ & 5 & 65 & Low & $\oplus$ \\
\hline All-cause mortality $[29]^{\mathrm{b}}$ & IFG/IGT: WHO & - & $1.07(0.75,1.52)$ & 2 & 0 & Low & $\oplus$ \\
\hline All-cause mortality $[29]^{\mathrm{b}}$ & IGT: ADA/WHO & - & $1.34(0.94,1.93)$ & 3 & 0 & Low & $\oplus$ \\
\hline All-cause mortality [29] ${ }^{\mathrm{b}}$ & IFG/IGT: ADA & & $1.62(0.96,2.73)$ & 2 & 0 & Low & $\oplus$ \\
\hline All-cause mortality $[29]^{\mathrm{b}}$ & $\mathrm{HbA}_{1 \mathrm{c}}: \mathrm{ADA}$ & & $2.30(0.56,9.41)$ & 2 & 77 & Low & $\oplus$ \\
\hline Long-term all-cause mortality [32] ${ }^{\mathrm{c}}$ & $\mathrm{HbA}_{1 \mathrm{c}}: \mathrm{ADA}$ & $-\infty$ & $1.18(0.92,1.50)$ & 3 & 0 & Unclear & $\oplus$ \\
\hline Short-term all-cause mortality $[32]^{\mathrm{c}}$ & $\mathrm{HbA}_{1 \mathrm{C}}: \mathrm{ADA}$ & & $1.00(0.69,1.44)$ & 2 & 0 & Unclear & $\oplus$ \\
\hline Stroke mortality [33] & IFG/IGT: ADA/WHO & & $1.40(0.68,2.91)$ & 4 & 65 & High & $\oplus$ \\
\hline Stroke mortality [33] ${ }^{\mathrm{d}}$ & IFG: WHO & & $1.64(0.67,4.01)$ & 2 & 38 & High & $\oplus$ \\
\hline Stroke mortality [33] ${ }^{d}$ & $\mathrm{HbA}_{1 \mathrm{c}}: \mathrm{ADA}$ & & $1.28(0.30,5.51)$ & 2 & 81 & High & $\oplus$ \\
\hline \multicolumn{8}{|l|}{ CVD outcomes and CV mortality } \\
\hline CV events [29] ${ }^{\mathrm{b}}$ & IFG: ADA & - & $1.33(1.02,1.75)$ & 6 & 81 & Low & $\oplus \oplus \oplus$ \\
\hline CV events [29] & IGT: ADA/WHO & $-\infty$ & $1.52(1.24,1.85)$ & 6 & 0 & Low & $\oplus \oplus \oplus$ \\
\hline CV events [29] ${ }^{\mathrm{b}}$ & IFG: WHO & & $1.49(0.99,2.24)$ & 5 & 83 & Low & $\oplus \oplus$ \\
\hline CV events [29] ${ }^{\mathrm{b}}$ & $\mathrm{HbA}_{1 \mathrm{c}}: \mathrm{ADA}$ & $-\infty$ & $1.24(1.05,1.48)$ & 4 & 0 & Low & $\oplus \oplus$ \\
\hline CV events [29] ${ }^{\mathrm{b}}$ & IFG/IGT/HbA $1 \mathrm{c}:$ ADA & 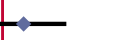 & $1.16(0.86,1.57)$ & 2 & 0 & Low & $\oplus$ \\
\hline CV events [29] ${ }^{\mathrm{b}}$ & $\mathrm{IFG} / \mathrm{HbA}_{1 \mathrm{c}}: \mathrm{ADA}$ & 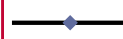 & $1.61(1.07,2.43)$ & 2 & 2 & Low & $\oplus \oplus$ \\
\hline $\mathrm{CHD}[29]^{\mathrm{b}}$ & IGT: ADA/WHO & $+\infty$ & $1.14(0.84,1.54)$ & 3 & 0 & Low & $\oplus$ \\
\hline $\mathrm{CHD}[29]^{\mathrm{b}}$ & IFG: ADA & $t \rightarrow$ & $1.10(0.92,1.30)$ & 2 & 0 & Low & $\oplus$ \\
\hline $\mathrm{CHD}[29]^{\mathrm{b}}$ & IFG: WHO & $-\infty$ & $1.24(0.99,1.56)$ & 2 & 0 & Low & $\oplus$ \\
\hline $\mathrm{CHD}[29]^{\mathrm{b}}$ & $\mathrm{HbA1C}: \mathrm{ADA}$ & - & $1.16(0.65,2.05)$ & 2 & 0 & Low & $\oplus$ \\
\hline Stroke $[29]^{\mathrm{b}}$ & IFG: ADA & - & $0.99(0.63,1.54)$ & 2 & 62 & Low & $\oplus$ \\
\hline Stroke $[33]^{\mathrm{d}}$ & IFG/IGT: ADA/WHO & $\longrightarrow$ & $1.45(0.98,2.14)$ & 3 & 32 & High & $\oplus$ \\
\hline Stroke $[33]^{\mathrm{d}}$ & IFG: WHO & 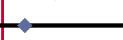 & $1.17(0.55,2.48)$ & 2 & 65 & High & $\oplus$ \\
\hline Poor outcome of stroke $[33]^{\mathrm{d}, \mathrm{e}}$ & IFG/IGT/HbA $1 \mathrm{C}: \mathrm{ADA} / \mathrm{WHO}$ & - & $1.41(1.01,1.97)$ & 5 & 56 & High & $\oplus \oplus$ \\
\hline Poor outcome of stroke $[33]^{\mathrm{d}, \mathrm{e}}$ & IFG: WHO & $\longrightarrow$ & $1.41(1.05,1.90)$ & 2 & 30 & High & $\oplus \oplus$ \\
\hline MACE $[31]^{c}$ & IFG/IGT/HbA $1 \mathrm{C}: \mathrm{ADA} / \mathrm{WHO}$ & $\rightarrow-$ & $1.41(1.14,1.75)$ & 10 & 31 & High & $\oplus \oplus$ \\
\hline MACE [31] & IGT: ADA/WHO & $\longrightarrow$ & $1.62(1.07,2.46)$ & 2 & 0 & High & $\oplus \oplus$ \\
\hline MACE $[31]^{\mathrm{c}}$ & $\mathrm{HbA}_{1 \mathrm{c}}: \mathrm{ADA}$ & & $1.38(0.88,2.17)$ & 3 & 63 & High & $\oplus$ \\
\hline \multicolumn{8}{|l|}{ 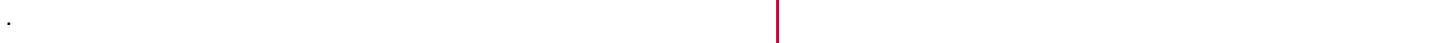 } \\
\hline & $\begin{array}{l}1 \\
0.5\end{array}$ & 2 & & & & & \\
\hline
\end{tabular}

Fig. 3 Associations between prediabetes and risk of CVD, cardiovascular mortality and overall mortality among patients with pre-existing diseases. anterpretation of the certainty of evidence is denoted by crossed circles: four symbols, high; three symbols, moderate; two symbols, low; and one symbol, very low. ${ }^{b}$ Patients with atherosclerotic CVD. ${ }^{c}$ Patients after percutaneous coronary intervention. ${ }^{\mathrm{d}}$ Patients with history of stroke/transient ischaemic attack. ${ }^{\text {P}}$ Poor outcome of stroke defined as degree of disability or dependence in the daily activities of people who have suffered a stroke or other causes of neurological disability. Boldface indicates that the 95\% CI does not include the null value and findings are precisely estimated. $\mathrm{CoE}$, certainty of evidence; $\mathrm{CV}$, cardiovascular; $N$, number of primary studies; MACE, major adverse cardiac events; RoB: Risk of bias dose-response gradient can lead to upgrading. The specific reasons for downgrading are provided with the results. The overall rating categorises the certainty of evidence into high, moderate, low or very low. High and moderate certainty in evidence means that it is very likely or probable that the true effect lies close to the estimated finding, and low or very low means that we have little or very little confidence in the finding.

\section{Results}

Out of the 4807 identified articles, 126 full-texts were assessed for eligibility, and finally, 16 articles were included in this umbrella review (Fig. 1) [7-10, 23-34]. A detailed list of the excluded articles and the reasons for their exclusion is displayed in ESM Table 3. Out of the included articles, 13 focused on individuals from the general population [7-10, 23-30, 34] and four on specific patient groups, including those with CVD [29], patients after percutaneous coronary intervention $[31,32]$ or in individuals with a history of stroke/transient ischaemic attack [33]. The 16 articles comprised 95 meta-analyses. Figures 2 and 3 and ESM Table 4 list all outcomes for which associations with prediabetes were identified.

The number of the included primary studies in the metaanalyses ranged from two to 25 , and these studies were conducted in the USA, Europe, Asia and Oceania. Prediabetes was defined by using different classifications and cut-off values for IFG, IGT and $\mathrm{HbA}_{1 \mathrm{c}}$ defined by the 
ADA or WHO. A detailed description of the meta-analyses is shown in ESM Table 4. Most of the primary studies adjusted for several important confounders (age, sex, BMI/waist circumference, smoking, BP/treatment of hypertension and blood lipids/treatment of hyperlipidaemia) (ESM Table 5).

The methodological quality was rated as low risk of bias for four reports [7, 8, 29, 34] and high risk of bias for the remaining reports. Major sources of bias were as follows: an unclear definition of prediabetes; the use of filters in the search (e.g. restricted to human studies); lack of clarity regarding whether screening of the studies, data extraction and/or assessment of the risk of bias of the studies were conducted independently by at least two investigators; and complete lack of the assessment of risk of bias and/or its discussion. A detailed description is shown in ESM Table 6.

Figure 2 summarises the SHRs with $95 \%$ CIs of each identified meta-analysis of data for the general population. Whenever possible, the meta-analyses are shown separately by the different definitions of prediabetes (IFG, IGT or $\mathrm{HbA}_{1 \mathrm{c}}$ with cut-offs provided by the ADA or WHO). There was moderate certainty of evidence for an association between prediabetes (IFG defined by the ADA and WHO and/or IGT) and increased risk of all-cause mortality, cardiovascular events (incidence and mortality combined), CVD mortality, CVD incidence, CHD, stroke, heart failure, atrial fibrillation, chronic kidney disease, total cancer, total liver cancer (liver and intrahepatic bile ducts), hepatocellular carcinoma, breast cancer and all-cause dementia (ESM Table 7). The relative risk of the associations ranged from 6 to $101 \%$ of these outcomes, with SHRs $(95 \% \mathrm{CI})$ of $1.06(1.01,1.11)$ for stroke to $2.01(1.45,2.79)$ for hepatocellular carcinoma. For allcause mortality and CVD, the relative risk was up to $39 \%$ higher in individuals with prediabetes compared with individuals within the normal glucose range. Of note, one metaanalysis did not detect an association between prediabetes classified according to IFG-ADA criteria and chronic kidney disease (low certainty of evidence). However, this metaanalysis applied a specific selection criterion and excluded studies on participants who developed diabetes after baseline [30], while in all other meta-analyses, including the other meta-analyses on chronic kidney disease [8], studies were only excluded when participants had diabetes at baseline.

Prediabetes was further associated with increased risk of sudden cardiac death, stomach and colorectal cancer combined, pancreatic cancer, Alzheimer's dementia and vascular dementia (Fig. 2), but certainty of evidence was low or very low (ESM Table 7). There were no (or imprecise) associations between prediabetes and bronchus and lung cancer, prostate cancer, kidney cancer, depressive symptoms and cognitive impairment (low or very low certainty of evidence).

Figure 3 summarises the SHRs with the corresponding 95\% CIs of each identified meta-analysis among specific patient groups. For patients with previous CVD, prediabetes (IFG-ADA) was associated with risk of all-cause mortality (low certainty of evidence) and with increased risk of further cardiovascular events (IFG-ADA, IGT, moderate certainty of evidence, and $\mathrm{HbA}_{1 \mathrm{c}}$, low certainty of evidence) (ESM Table 7). There was low or very low certainty of evidence that prediabetes was associated with increased risk of stroke and poor outcomes in patients with history of stroke/transient ischaemic attack, or with major adverse cardiac events in patients with previous percutaneous coronary intervention. For stroke mortality, the direction of the meta-analyses for both patient groups also pointed to an increased SHR, although the $95 \%$ CIs were imprecisely estimated.

The heterogeneity in the included meta-analyses varied, with $I^{2}$ between 0 and $83 \%$. The variance of the true effect sizes $\left(\tau^{2}\right)$ ranged between 0 and 0.917 (ESM Table 4), but $p$ values were mostly $<0.05$. For ten associations, the $95 \%$ prediction intervals excluded unity (outcomes, risk of allcause mortality; cardiovascular events; CHD; stroke; heart failure; and pancreatic cancer). This result means that the true effect sizes in future studies can be expected to point in the same direction as in the meta-analyses presented here; however, for most of the associations, smaller or null findings could be possible in other samples.

For meta-analyses with more than ten studies, there was no indication of small study effects/publication bias for most of the associations (ESM Fig. 1), with the exception of the association between IFG-ADA and IGT regarding cardiovascular events and for IFG-WHO regarding CHD. For these metaanalyses, there was indication of small study effects according to Egger's test $(p<0.1)$ and indication for missing of small studies in the funnel plots.

In general, there was indication for stronger associations for IGT than for IFG or $\mathrm{HbA}_{1 \mathrm{c}}$ regarding several outcomes (e.g. risk of all-cause mortality, cardiovascular events, CHD and stroke). However, the 95\% CIs mostly overlapped, and thus, a clear gradient was observed only for risk of all-cause mortality.

\section{Discussion}

Prediabetes was associated with a higher risk of all-cause mortality and incident cardiovascular events, CHD, stroke, heart failure, atrial fibrillation, chronic kidney disease, total cancer, total liver cancer, hepatocellular carcinoma, breast cancer and all-cause dementia, with moderate certainty of evidence, in meta-analyses including individuals from the general population and with worse prognosis in patients with previous cardiovascular problems. Summary effect estimates were higher for IGT-defined prediabetes and weaker for $\mathrm{HbA}_{1 \mathrm{c}}$-defined prediabetes compared with IFG-defined prediabetes; this was most pronounced for risk of all-cause 
mortality as an outcome. No associations were observed between prediabetes and incident depressive symptoms, cognitive impairment or some specific cancers. The risk of bias was high for most meta-analyses included in this umbrella review, showing that authors of future meta-analyses should adhere to established recommendations regarding the conduct and reporting of systematic reviews and meta-analyses and should assess and discuss the risk of bias of primary studies. No eligible meta-analyses could be identified for the incidence of neuropathy and retinopathy.

All-cause mortality Prediabetes defined by IFG or IGT, but not prediabetes defined using $\mathrm{HbA}_{1 \mathrm{c}}$, was associated with an 8 $25 \%$ increased risk of all-cause mortality. In comparison, the risk for all-cause death was between 60 and $100 \%$ higher in people with diabetes than in people without diabetes in recent studies $[35,36]$. The summary effect size was higher for prediabetes defined by IGT than for that defined by IFG (without overlapping 95\% CIs between IGT and IFG/WHO) or for $\mathrm{HbA}_{1 \mathrm{c}}$-defined prediabetes, pointing towards the superior utility of the OGTT in the identification of high-risk individuals.

Cardiovascular outcomes Prediabetes defined by IFG, IGT and $\mathrm{HbA}_{1 \mathrm{c}}$ was also positively associated with risk of $\mathrm{CV}$ mortality and multiple CV outcomes such as incident CVD, CHD, stroke, heart failure and atrial fibrillation. The SHRs for prediabetes and risk of $\mathrm{CV}$ mortality ranged from 1.2 to 1.3 . These data can be compared with CVD-related mortality data from the USA from 2000 to 2015 showing risk for people with vs without diabetes of 1.8-2.1 [35, 37]. Thus, the excess risk conferred by prediabetes appears to be less than one-third when compared with the risk related to diabetes. As the identified meta-analyses on stroke did not distinguish between ischaemic and haemorrhagic stroke [23, 29], we also restricted our analysis to stroke as a combined outcome. Potential differences in the associations between prediabetes and both stroke types need studying further.

Microvascular outcomes One meta-analysis on prediabetes and chronic kidney disease was available, with an SHR of 1.10 and 1.25 depending on the underlying definition of IFG. This result can be compared with an approximate threefold higher risk of chronic kidney disease in people with diabetes than in those without diabetes [38]. This suggests that prediabetes, at least when defined by IFG, may be a substantially weaker risk factor for chronic kidney disease than diabetes.

Our study protocol also included diabetic retinopathy, macular oedema, vitreous haemorrhage and diabetes-related blindness as well as peripheral and autonomic (poly)neuropathy, diabetic foot ulcers and amputations as outcomes of interest, but our systematic literature search did not identify any relevant meta-analyses. Given that the prevalence of retinopathy was initially used to refine threshold levels of fasting plasma glucose, $2 \mathrm{~h}$ plasma glucose and $\mathrm{HbA}_{1 \mathrm{c}}$ for the diagnosis of diabetes [39], it is not surprising that the association between prediabetes and retinopathy has been reported to be weak [40]. In contrast, evidence is accumulating for the presence of peripheral and cardiac autonomic neuropathy in people with prediabetes [41-44], so this field needs further prospective studies to quantify this relationship.

Cancer Depending on how prediabetes was defined, the SHR for the association between prediabetes and total cancer incidence ranged from 1.11 to 1.25 . In comparison, total cancer incidence and cancer mortality were 1.1- to 1.4-fold higher in people with diabetes than in those without diabetes [45]. Associations with moderate certainty of evidence between prediabetes and site-specific cancers appeared stronger for total liver cancer (twofold higher) and hepatocellular carcinoma (SHR 1.44) compared with other cancers. An umbrella review on type 2 diabetes and cancer incidence found varying associations with site-specific cancers, but in line with our study, there was evidence for a link with incident liver and colorectal cancer [35, 45, 46]. Overall, effect estimates for prediabetes and diabetes appear similar with cancer as an outcome.

Mental health People with prediabetes showed an 18-47\% increased risk for dementia (all-cause, Alzheimer's or vascular) than people without prediabetes. In people with diabetes, the excess risk has been estimated to be $60-130 \%$ compared with people without diabetes, depending on the type of dementia and the sex of the individual [47]. In contrast, no associations were found between prediabetes and cognitive impairment or depressive symptoms, although increased risks for both conditions have been reported for people with diabetes $[9,28,48]$. It is possible that more pronounced hyperglycaemia is required for the development of depressive symptoms or clinical depression than that found in prediabetes, whereas the discrepancies of the data between dementia and cognitive impairment are more challenging to interpret. However, it should be noted that the meta-analyses were based on small numbers of primary studies, and future studies might add evidence to these findings.

Comparison between different prediabetes definitions In the meta-analyses included in this report, different definitions for prediabetes were used, and for some outcomes, meta-analyses for multiple definitions were conducted separately whenever possible. In general, the SHRs were in the same direction independently of the used prediabetes definition. A clear difference was seen for risk of all-cause mortality, CHD and stroke, with the strongest association for IGTdefined prediabetes and an intermediate effect size for 
IFG-defined prediabetes [7, 29]. For the other outcomes, overlapping $95 \%$ CIs of the different prediabetes definitions precluded any firm conclusions. Of note, all metaanalyses on prediabetes defined by $\mathrm{HbA}_{1 \mathrm{c}}$ included $\leq 5$ primary studies, which might explain this lack of evidence. However, $\mathrm{HbA}_{1 \mathrm{c}}$ shows only relatively weak correlations with fasting and $2 \mathrm{~h}$ plasma glucose in people without type 2 diabetes, meaning that its main determinants in people without diabetes are not glucose-related and are linked to different underlying (patho)physiological processes [49].

Risk of bias and certainty of evidence Based on the ROBIS tool, all except four of the reports identified for this umbrella review were graded as being at high risk of bias. Major potential sources of bias comprised unclear prediabetes definitions and issues with search strategy, data extraction and/or assessment and discussion of the risk of bias of the primary studies. Although ROBIS is a validated Cochrane tool with which to evaluate the risk of bias in such studies, it is limited by the fact that it only distinguishes between high and low risk of bias. However, it has to be emphasised that authors of systematic reviews and meta-analyses should adhere to established guidelines regarding the conduct and reporting of their reviews. In addition, risk of bias evaluation of the primary studies is a crucial aspect in grading of the evidence and needs to be included in meta-analyses.

According to the GRADE tool, cohort studies start with a low certainty of evidence because of the nature of the observational study design, implying the possibility of residual or unknown confounding. We checked the confounders included in all primary studies, and in most of them, important known confounders were considered. Furthermore, in our umbrella review, we upgraded the certainty of evidence when a doseresponse gradient was expected. Therefore, we assumed a dose-response gradient in the following situations: when associations were stronger for IFG-defined prediabetes when IFG was defined by the WHO compared with the ADA (different cut-off values); when associations were stronger for IGT- than for IFG-defined prediabetes; or when the original meta-analysis conducted a dose-response meta-analysis. However, we did not investigate dose-response metaanalyses by ourselves, since we investigated prediabetes as a binary variable (yes/no). Moreover, several of the associations in our report were graded as having a low or very low certainty of evidence, showing that more studies are needed to strengthen the evidence.

Strengths and limitations This umbrella review had several strengths. It provides the first systematic overview of the evidence from meta-analyses on the association between prediabetes and the incidence of 'diabetes-related' complications. We extracted data from primary studies and recalculated the SHR after excluding studies that were not in accordance with our a priori defined prediabetes categories. Whenever possible, we calculated effect estimates for all available prediabetes definitions. Risk of bias and certainty of evidence were assessed using the established ROBIS and GRADE tools, respectively. Finally, we identified gaps in the literature pointing towards future research needs.

Our umbrella review also had limitations, mostly related to the included meta-analyses or primary studies. First, the primary studies included in the identified meta-analyses had an observational design so that residual confounding cannot be ruled out. Second, study participants with prediabetes may have developed type 2 diabetes during the follow-up period, and this was usually not accounted for. Third, for most of the meta-analyses included in this umbrella review, risk of bias was high, and the certainty of evidence for some associations was low or very low, so that future high-quality studies are needed to corroborate our findings. Fourth, we did not explore heterogeneity in subgroups or sensitivity analyses, although whenever possible we differentiated between different prediabetes definitions. Finally, publication bias could not be assessed for most associations because of the small number of primary studies contributing to many of the identified metaanalyses.

Conclusions Prediabetes was associated with a higher relative risk of all-cause mortality and higher incidences of $\mathrm{CV}$ events, CHD, stroke, heart failure, atrial fibrillation, chronic kidney disease, total cancer, liver cancer, hepatocellular carcinoma, breast cancer and all-cause dementia with moderate certainty of evidence. Effect estimates were lower than comparable effect estimates for type 2 diabetes as exposure, suggesting a doseresponse gradient in the relationship with complications. For risk of all-cause mortality, the association was stronger for IGT- than for IFG- or $\mathrm{HbA}_{1 \mathrm{c}}$-defined prediabetes, which points to towards the superior utility of the OGTT in the identification of high-risk individuals. Of note, no meta-analyses were found for associations between prediabetes and common diabetes-related complications such as neuropathy and retinopathy. The consistency of the results, the high global prevalence of prediabetes and the possibility of improving glucose metabolism and related risk factors in people with prediabetes using lifestyle interventions should lead to intensified research in this field. Given the identified research gaps and high risk of bias of the metaanalyses included in this umbrella review, higherquality studies (both primary studies and metaanalyses) on the associations between prediabetes and multiple health outcomes are required to more robustly estimate the potential medical and cost-related benefits of intervention measures at this stage. 
Supplementary Information The online version contains peer-reviewed but unedited supplementary material available at https://doi.org/10.1007/ s00125-021-05592-3.

Authors' relationships and activities WR reports receiving consulting fees for attending educational sessions or advisory boards from AstraZeneca, Boehringer Ingelheim and NovoNordisk and institutional research grants from NovoNordisk outside the submitted work. MR is or has been on the scientific advisory boards of Allergan, AstraZeneca, Bristol-Myers Squibb, Eli Lilly, Gilead Sciences, Inventiva, Intercept Pharma, Novartis, NovoNordisk, Servier Laboratories, Target Pharmasolutions and Terra Firma and receives investigator-initiated support from Boehringer Ingelheim, Nutricia/Danone and SanofiAventis outside the submitted work. $\mathrm{CH}$ received honoraria from Lilly and Sanofi and a research grant from Sanofi outside the submitted work. $\mathrm{CH}$ is a member of the editorial board of Diabetologia. All other authors declare that there are no relationships or activities that might bias, or be perceived to bias, their work.

Data availability All data generated or analysed during this study are included in this published article (and its supplementary information files).

Funding Open Access funding enabled and organized by Projekt DEAL. The German Diabetes Center (DDZ) is funded by the German Federal Ministry of Health and the Ministry of Science and Culture of the State North Rhine-Westphalia. This study was supported in part by a grant from the German Federal Ministry of Education and Research to the German Center for Diabetes Research (DZD). The funders had no role in study design or data collection, analysis and interpretation.

Contribution statement SS and $\mathrm{CH}$ designed the study and wrote the first draft of the manuscript. SS, JB and $\mathrm{CH}$ performed the literature search and literature screening. JB, MN, AL and HM extracted data. $\mathrm{JB}, \mathrm{MN}$ and SS assessed the risk of bias of the meta-analyses. MN and SS assessed the certainty of evidence of the associations. MN performed statistical analyses. All authors contributed to data acquisition, data interpretation, revision of manuscript drafts and have read and approved the final manuscript. SS is the guarantor of this work and, as such, had full access to all the data in the study and take responsibility for the integrity of the data and the accuracy of the data analysis.

Open Access This article is licensed under a Creative Commons Attribution 4.0 International License, which permits use, sharing, adaptation, distribution and reproduction in any medium or format, as long as you give appropriate credit to the original author(s) and the source, provide a link to the Creative Commons licence, and indicate if changes were made. The images or other third party material in this article are included in the article's Creative Commons licence, unless indicated otherwise in a credit line to the material. If material is not included in the article's Creative Commons licence and your intended use is not permitted by statutory regulation or exceeds the permitted use, you will need to obtain permission directly from the copyright holder. To view a copy of this licence, visit http://creativecommons.org/licenses/by/4.0/.

\section{References}

1. American Diabetes Association (2020) 2. Classification and diagnosis of diabetes: standards of medical Care in Diabetes-2020. Diabetes Care 43(Suppl 1):S14-S31. https://doi.org/10.2337/ dc20-S002
2. International Diabetes Federation (2019) Diabetes Atlas, 9th edn. Available from: https://www.diabetesatlas.org. Accessed 22 Feb 2021

3. Wang L, Gao P, Zhang M et al (2017) Prevalence and ethnic pattern of diabetes and prediabetes in China in 2013. JAMA 317(24): 2515-2523. https://doi.org/10.1001/jama.2017.7596

4. Richter B, Hemmingsen B, Metzendorf MI, Takwoingi Y (2018) Development of type 2 diabetes mellitus in people with intermediate hyperglycaemia. Cochrane Database Syst Rev 10:CD012661. https://doi.org/10.1002/14651858.CD012661.pub2

5. Roden M, Shulman GI (2019) The integrative biology of type 2 diabetes. Nature 576(7785):51-60. https://doi.org/10.1038/s41586019-1797-8

6. Tabak AG, Herder C, Rathmann W, Brunner EJ, Kivimaki M (2012) Prediabetes: a high-risk state for diabetes development. Lancet 379(9833):2279-2290. https://doi.org/10.1016/S01406736(12)60283-9

7. Huang Y, Cai X, Mai W, Li M, Hu Y (2016) Association between prediabetes and risk of cardiovascular disease and all cause mortality: systematic review and meta-analysis. BMJ 355:i5953. https:// doi.org/10.1136/bmj.i5953

8. Echouffo-Tcheugui JB, Venkat Narayan KM, Weisman D, Golden SH, Jaar BG (2016) Association between prediabetes and risk of chronic kidney disease: a systematic review and meta-analysis. Diabet Med 33(12):1615-1624. https://doi.org/10.1111/dme.13113

9. Xue M, Xu W, Ou YN et al (2019) Diabetes mellitus and risks of cognitive impairment and dementia: a systematic review and metaanalysis of 144 prospective studies. Ageing Res Rev 55:100944. https://doi.org/10.1016/j.arr.2019.100944

10. Huang Y, Cai X, Qiu M et al (2014) Prediabetes and the risk of cancer: a meta-analysis. Diabetologia 57(11):2261-2269. https:// doi.org/10.1007/s00125-014-3361-2

11. Palladino R, Tabak AG, Khunti K et al (2020) Association between pre-diabetes and microvascular and macrovascular disease in newly diagnosed type 2 diabetes. BMJ Open Diabetes Res Care 8(1). https://doi.org/10.1136/bmjdrc-2019-001061

12. Haw JS, Galaviz KI, Straus AN et al (2017) Long-term sustainability of diabetes prevention approaches: a systematic review and Meta-analysis of randomized clinical trials. JAMA Intern Med 177(12):1808-1817. https://doi.org/10.1001/jamainternmed.2017.6040

13. Kowall B, Rathmann W, Heier M et al (2012) Impact of weight and weight change on normalization of prediabetes and on persistence of normal glucose tolerance in an older population: the KORA S4/ F4 study. Int J Obes 36(6):826-833. https://doi.org/10.1038/ijo. 2011.161

14. Gong Q, Zhang P, Wang J et al (2019) Morbidity and mortality after lifestyle intervention for people with impaired glucose tolerance: 30-year results of the Da Qing diabetes prevention outcome study. Lancet Diabetes Endocrinol 7(6):452-461. https://doi.org/ 10.1016/S2213-8587(19)30093-2

15. Nathan DM, Bennett PH, Crandall JP et al (2019) Does diabetes prevention translate into reduced long-term vascular complications of diabetes? Diabetologia 62(8):1319-1328. https://doi.org/10. 1007/s00125-019-4928-8

16. Aromataris E, Fernandez R, Godfrey CM, Holly C, Khalil H, Tungpunkom P (2015) Summarizing systematic reviews: methodological development, conduct and reporting of an umbrella review approach. Int J Evid Based Healthc 13(3):132-140. https://doi.org/ 10.1097/XEB.0000000000000055

17. Moher D, Liberati A, Tetzlaff J, Altman DG, PRISMA Group (2009) Preferred reporting items for systematic reviews and metaanalyses: the PRISMA statement. BMJ 339:b2535. https://doi.org/ 10.1136/bmj.b2535

18. Bougioukas KI, Bouras E, Apostolidou-Kiouti F, Kokkali S, Arvanitidou M, Haidich AB (2019) Reporting guidelines on how to write a complete and transparent abstract for overviews of 
systematic reviews of health care interventions. J Clin Epidemiol 106:70-79. https://doi.org/10.1016/j.jclinepi.2018.10.005

19. Whiting P, Savovic J, Higgins JP et al (2016) ROBIS: a new tool to assess risk of bias in systematic reviews was developed. J Clin Epidemiol 69:225-234. https://doi.org/10.1016/j.jclinepi.2015.06.005

20. DerSimonian R, Laird N (1986) Meta-analysis in clinical trials. Control Clin Trials 7(3):177-188. https://doi.org/10.1016/01972456(86)90046-2

21. Higgins JPT, Green S (2011) Cochrane Handbook for Systematic Reviews of Interventions Version 5.1.0 [updated March 2011] The Cochrane Collaboration: available from www.handbook.Cochrane. org. Accessed 1 Mar 2021

22. Zhang Y, Akl EA, Schunemann HJ (2018) Using systematic reviews in guideline development: the GRADE approach. Res Synth Methods. https://doi.org/10.1002/jrsm.1313

23. Mitsios JP, Ekinci EI, Mitsios GP, Churilov L, Thijs V (2018) Relationship between glycated hemoglobin and stroke risk: a systematic review and Meta-analysis. J Am Heart Assoc 7(11):110. https://doi.org/10.1161/JAHA.117.007858

24. Aune D, Feng T, Schlesinger S, Janszky I, Norat T, Riboli E (2018) Diabetes mellitus, blood glucose and the risk of atrial fibrillation: a systematic review and meta-analysis of cohort studies. J Diabetes Complicat 32(5):501-511. https://doi.org/10.1016/j.jdiacomp. 2018.02.004

25. Aune D, Schlesinger S, Norat T, Riboli E (2018) Diabetes mellitus and the risk of sudden cardiac death: a systematic review and metaanalysis of prospective studies. Nutr Metab Cardiovasc Dis 28(6): 543-556. https://doi.org/10.1016/j.numecd.2018.02.011

26. Xu WG, Qian YF, Wu J (2017) The effect of prediabetes on hepatocellular carcinoma risk: a systematic review and meta-analysis. Minerva Med 108(2):185-190. https://doi.org/10.23736/s00264806.16.04601-2

27. Fu DX, Cui HB, Guo NN, Su N, Xu JX, Wang GY (2016) Prediabetes and the risk of pancreatic cancer: a meta-analysis. Int J Clin Exp Med 9(10):19474-19479

28. Tong A, Wang X, Li F, Xu F, Li Q, Zhang F (2016) Risk of depressive symptoms associated with impaired glucose metabolism, newly diagnosed diabetes, and previously diagnosed diabetes: a meta-analysis of prospective cohort studies. Acta Diabetol 53(4): 589-598. https://doi.org/10.1007/s00592-016-0845-1

29. Cai X, Zhang Y, Li M et al (2020) Association between prediabetes and risk of all cause mortality and cardiovascular disease: updated meta-analysis. BMJ 370:m2297. https://doi.org/10.1136/bmj. m2297

30. Mutie PM, Pomares-Millan H, Atabaki-Pasdar N et al (2020) An investigation of causal relationships between prediabetes and vascular complications. Nat Commun 11(1):4592. https://doi.org/ 10.1038/s41467-020-18386-9

31. Zhao Y, Guo M, Shi G (2020) Prediabetes predicts adverse cardiovascular outcomes after percutaneous coronary intervention: a meta-analysis. Biosci Rep 40(1):BSR20193130. https://doi.org/10. 1042/bsr20193130

32. Li Y, Li XW, Zhang YH et al (2020) Prognostic significance of the hemoglobin A1c level in non-diabetic patients undergoing percutaneous coronary intervention: a meta-analysis. Chin Med J 133(18): 2229-2235. https://doi.org/10.1097/cm9.0000000000001029

33. Pan Y, Chen W, Wang Y (2019) Prediabetes and outcome of ischemic stroke or transient ischemic attack: a systematic review and Meta-analysis. J Stroke Cerebrovasc Dis 28(3):683-692. https:// doi.org/10.1016/j.jstrokecerebrovasdis.2018.11.008

34. Cai X, Liu X, Sun L et al (2021) Prediabetes and the risk of heart failure: a meta-analysis. Diabetes Obes Metab 21(8):1746-1753. https://doi.org/10.1111/dom.14388

35. Gregg EW, Cheng YJ, Srinivasan M et al (2018) Trends in causespecific mortality among adults with and without diagnosed diabetes in the USA: an epidemiological analysis of linked national survey and vital statistics data. Lancet 391(10138):2430-2440. https://doi.org/10.1016/S0140-6736(18)30314-3

36. Wang Y, O'Neil A, Jiao Y et al (2019) Sex differences in the association between diabetes and risk of cardiovascular disease, cancer, and all-cause and cause-specific mortality: a systematic review and meta-analysis of 5,162,654 participants. BMC Med 17(1):136. https://doi.org/10.1186/s12916-019-1355-0

37. Cheng YJ, Imperatore G, Geiss LS et al (2018) Trends and disparities in cardiovascular mortality among U.S. adults with and without self-reported diabetes, 1988-2015. Diabetes Care 41(11):23062315. https://doi.org/10.2337/dc18-0831

38. Shen Y, Cai R, Sun J et al (2017) Diabetes mellitus as a risk factor for incident chronic kidney disease and end-stage renal disease in women compared with men: a systematic review and meta-analysis. Endocrine 55(1):66-76. https://doi.org/10.1007/s12020-016-10146

39. Colagiuri S, Lee CM, Wong TY et al (2011) Glycemic thresholds for diabetes-specific retinopathy: implications for diagnostic criteria for diabetes. Diabetes Care 34(1):145-150. https://doi.org/10.2337/ dc10-1206

40. Lamparter J, Raum P, Pfeiffer N et al (2014) Prevalence and associations of diabetic retinopathy in a large cohort of prediabetic subjects: the Gutenberg health study. J Diabetes Complicat 28(4): 482-487. https://doi.org/10.1016/j.jdiacomp.2014.02.008

41. Herder C, Roden M, Ziegler D (2019) Novel insights into sensorimotor and cardiovascular autonomic neuropathy from recent-onset diabetes and population-based cohorts. Trends Endocrinol Metab 30(5):286-298. https://doi.org/10.1016/j.tem.2019.02.007

42. Bonhöf GJ, Herder C, Strom A, Papanas N, Roden M, Ziegler D (2019) Emerging biomarkers, tools, and treatments for diabetic polyneuropathy. Endocr Rev 40(1):153-192. https://doi.org/10. 1210/er.2018-00107

43. Eleftheriadou A, Williams S, Nevitt S et al (2021) The prevalence of cardiac autonomic neuropathy in prediabetes: a systematic review. Diabetologia 64(2):288-303. https://doi.org/10.1007/ s00125-020-05316-Z

44. Kirthi V, Perumbalath A, Brown E et al (2021) Prevalence of peripheral neuropathy in pre-diabetes: a systematic review. BMJ Open Diabetes Res Care 9(1):e002040. https://doi.org/10.1136/ bmjdrc-2020-002040

45. Tsilidis KK, Kasimis JC, Lopez DS, Ntzani EE, Ioannidis JP (2015) Type 2 diabetes and cancer: umbrella review of meta-analyses of observational studies. BMJ 350:g7607. https://doi.org/10.1136/ bmj.g7607

46. Chen Y, Wu F, Saito E et al (2017) Association between type 2 diabetes and risk of cancer mortality: a pooled analysis of over 771, 000 individuals in the Asia cohort consortium. Diabetologia 60(6): 1022-1032. https://doi.org/10.1007/s00125-017-4229-z

47. Chatterjee S, Peters SA, Woodward M et al (2016) Type 2 diabetes as a risk factor for dementia in women compared with men: a pooled analysis of 2.3 million people comprising more than 100 , 000 cases of dementia. Diabetes Care 39(2):300-307. https://doi. org $/ 10.2337 / \mathrm{dc} 15-1588$

48. Rotella F, Mannucci E (2013) Diabetes mellitus as a risk factor for depression. A meta-analysis of longitudinal studies. Diabetes Res Clin Pract 99(2):98-104. https://doi.org/10.1016/j.diabres.2012.11. 022

49. Fizelova M, Stancakova A, Lorenzo C et al (2015) Glycated hemoglobin levels are mostly dependent on nonglycemic parameters in 9398 Finnish men without diabetes. J Clin Endocrinol Metab 100(5):1989-1996. https://doi.org/10.1210/jc.2014-4121

Publisher's note Springer Nature remains neutral with regard to jurisdictional claims in published maps and institutional affiliations. 